\title{
Interconnections between the discursive framing of space-time and the interpretation of a collaborative task
}

\section{Ritella, Giuseppe}

2019-03

Ritella , G , Ligorio, M B \& Hakkarainen, K 2019 , ' Interconnections between the discursive framing of space-time and the interpretation of a collaborative task ' , Learning, Culture and Social Interaction, vol. 20 , pp. 45-57 . https://doi.org/10.1016/j.lcsi.2017.08.001

http://hdl.handle.net/10138/326682

https://doi.org/10.1016/j.Icsi.2017.08.001

cc_by_nc_nd

acceptedVersion

Downloaded from Helda, University of Helsinki institutional repository.

This is an electronic reprint of the original article.

This reprint may differ from the original in pagination and typographic detail.

Please cite the original version. 
This is a draft version for self-archiving: Ritella, G., Ligorio, M. B., \& Hakkarainen, K. (2019). Interconnections between the discursive framing of space-time and the interpretation of a collaborative task. Learning, Culture and Social Interaction, 20, 45-57.

Interconnections between the discursive framing of space-time and the interpretation of a collaborative task

\begin{abstract}
The purpose of the present study was to examine the discursive framing of space-time in the context of a collaborative task in a higher education course. We employed the concept of chronotope (Bakhtin, 1982), which was developed within the dialogical approach for a socio-cultural examination of space and time. We conducted participant observation at a media-design course in which students worked in groups to develop a project. We collected video-audio records of the students' activity and analysed qualitatively the students' interaction whenever we detected a link between the framing of space-time relations (chronotope) and the interpretation of the collaborative task. The analysis identified some aspects of chronotopes that appear tightly connected to the interpretation of the task: 1) the future-oriented envisioning of potential trajectories of engagement; 2) definitions of the time structure of the activity; 3) consideration of chronotopic constraints; 4) monitoring of the task; 5) focus on the present moment. Furthermore, we have found that the students had different assumptions concerning the space-time of the activity depending on their professional background. In sum, we argue that the framing of space-time is a further aspect of the context involved in the process of task interpretation, additional to the institutional aspects already identified by previous research.
\end{abstract}

Keywords: chronotope, task interpretation, CSCL, context 


\section{Introduction}

The purpose of the present study was to examine the discursive framing of space-time in the context of a collaborative task in a higher education course. To reach this aim we employed the concept of chronotope (Bakhtin, 1982), which was developed within the dialogical approach for a sociocultural examination of space and time. This concept, in our view, has great potential for enriching the understanding of social interaction during collaborative tasks supported by technology. In particular, we analyse the collaborative design of a product within a media design course, discussing how the discursive framing of space-time is intertwined with the students' interpretation of the collaborative task.

How participants negotiate a shared understanding of a task is crucial for collective action. This process involves multiple interpretations of the task, some of which are considered deviations from the task as assigned by the teachers. Disregarding the multiple interpretations of the task emerging in dialogical interaction might hinder attempts to understand how students relate to and engage in assigned tasks (Rajala \& Sannino, 2015). A few pioneering studies have revealed that some aspects of the context can play important roles in students' interpretations of tasks (Säljö \& Windhamm, 1993; Lantz-Anderson et al., 2009; Rajala \& Sannino, 2015). In particular, these studies show that the institutional context of schooling strongly influence how students interpret tasks. For example, presenting the same task during a lesson, in a one-to-one tutorial, or in an after-school club, results in different interpretations by the students (Newman et al., 1989). Nevertheless, it is unclear yet how the discursive framing of space-time contributes to this process.

In this paper, we consider the discursive framing of space-time as an intrinsic aspect of the context, and we analyse how this is intertwined with the building of a shared interpretation of the task. These processes are especially important when students engage in open-ended collaborative tasks requiring creative solutions to complex "ill-structured problems" (Jonassen, 2000), such as the design of a 
product, where the students have a high degree of freedom in defining both the scope and the organization of the activity.

In the next section, we will briefly discuss our conceptualization of chronotope for the present investigation. Then we will describe the context, methods and findings of a case study involving a media design course at a university of applied science. Finally, we discuss the findings and draw the conclusions.

\section{The concept of chronotope}

The concept of chronotope - crafted from the Greek words chronos and topos, meaning time and place/space - was used by Bakhtin (1981) to analyse the space-time relations that characterize a literary genre. Even though the literature using the concept is not extensive, recently researchers have found it useful to examine diverse aspects of social interaction and collaboration in educational settings. This literature is based on the assumption that space and time are interdependent social constructs rather than independent given realities (van Eljick \& Roth, 2010). Following Bakhtin, they consider spatial and temporal processes interdependent, when considered in analysing human action. Indeed, the analysis of activities and their contexts requires a co-ordinated understanding of space and time: different types of space for a given activity can entail different temporal relations and vice versa. For example, in a previous investigation on collaborative problem solving we have discussed that the movement of the participants around the computer and the arrangement of the virtual space was one of the aspects regulating the tempo of the activity (Ligorio \& Ritella, 2010). Given such interdependence between spatial and temporal relations, it is crucial to analyse space and time in a coordinated way.

Using the concept of chronotope can significantly enrich our understanding of social practices in educational settings (see, for example, Brown \& Renshaw, 2006; Renshaw, 2013). This concept is particularly relevant when technology is involved in social practices because the use of technology radically transforms space-time relations. The entire temporally layered flow of activity is 
transformed when different types of technology are used. The operation of an instrument requires the mastery of temporally layered procedures and practices that differ significantly according to the instrument (Norman, 1994). The use of technology also transforms spatial relations, for example, by means of virtual spaces that can be organised and manipulated in different ways, and by means of mobile technology, which makes information accessible regardless of location. Chronotopic analysis involves the examination of both the space-time organization of the material and technological environments in which social practices take place, and of the discursive framing of space-time involved in the organization of human experience.

\section{Analysing the discursive framing of space-time}

In this paper, we will contribute to the literature on chronotope by focusing on one specific aspect of space-time, namely how participants discursively frame the space and time of their activity while participating in a collaborative design task within a technologically rich environment. This process involves discourse regarding the organization of the workspace (both virtual and physical), the temporal organization of the activity, the coordination of individual actions, the time constraints perceived by the students, and so on. It partially overlaps with what Engle (2006) calls the framing of the context: when participants frame the context, they also define it in terms of the spaces that are relevant for the activity at a certain time and the temporal boundaries and time structure of the activity.

We consider the context of an activity as the outcome of a continuous process of social construction engaged in by participants, who embody meaning in the spaces and artefacts around them, according to the activity of the moment (Cole, 1996; Duranti \& Goodwin, 1992). In order to examine the results of such process of framing it is possible to think in terms of the emergence of multiple, heterogeneous and often overlapping spaces. For example, the physical spaces of a school such as classrooms, laboratories and even toilets might overlap with the socially organized spaces involving intimate, social and public zones, and with the multiple symbolic spaces of books, blackboards, computers, etc. The same classroom, then, can be examined as a physical space involving the measurement of the 
dimension and shape of the room, as a social space where some zones of the classroom are privileged for some kind of social interactions and not for others, and as a semiotic/symbolic space in terms of spatially ordered signs and symbols interpreted by the teachers and students. In this article, we examine how the students discursively frame different types of spaces while negotiating a shared understanding of the task. In addition, given the potential interdependence of space and time, we consider also temporal frames in the analysis, carrying out a coordinated analysis.

To investigate the temporal dimension of chronotope, we adopt the conceptualization of temporality defined by Valsiner (2011), who argues that at each point in time the future entails 'a variety of equally potential (not yet actualized) trajectories' (p. 142), some of which will never be actualized. These trajectories constitute the horizon of possibilities within which human action takes place, and they can be reflected on by the participants in any activity. In particular, Valsiner points to two types of temporality seldom investigated but crucial for understanding how people link the present to the future: "time as it could be" and "time as it should be". In our analysis of task interpretation, we discuss how these types of temporalities emerge from the students' discourse, and how they contribute to the actualization of certain trajectories and not others.

Following Bakhtin, we do not isolate temporality from spatiality; we consider them as inherently interdependent. This does not mean that they are always referred to in discourse together. The discursive framing of space-time is never complete nor comprehensive; the students' discourse contains references only to the space and time frames that support their situational (moment-tomoment) sense-making regarding the task at hand, as they are subjectively enacted, experienced and socially negotiated. Therefore, in some occasions it is possible that the participants might consider mainly space or mainly time. In these cases, the aspect which is not mentioned does not vanish; it remains implicit, taken for granted, as part of the invisible grounding of activities. Given the discursive nature of this analysis, we do not examine space and time as objective dimensions of activity. Rather, we analyse how the students frame them in discourse, and how such an 
intersubjective framing of space-time is involved in regulating the students' understanding of and engagement in the task. The discursive framing of space-time is especially important for collaborative design tasks because students negotiate with their peers and with teachers the space-time frames of the collaborative activity, and because working environments are often diversified and complex. When a large number of technological tools and physical, social and virtual spaces are available, their management is not trivial. A complex orchestration is needed to successfully organize the activity and coordinate individual efforts.

Our hypothesis is that such spatial and temporal frames might have an impact on the interpretation of the task. We consider this process as connected to the building of a joint problem space (JPS) (Roschelle \& Teasley, 1995) and to the object formation in collective activity (Engeström, 1999; Kirsten, 2002). We add a special focus on space-time framing, which is an aspect of the context that is underdeveloped in the literature.

\section{The study: Interpreting the task during a CSCL project course}

\subsection{Research questions}

As stated above, we focus on the social framing of space-time and on how this process is interdependent with students' interpretation of the task. To reach this aim, we analyse a higher education project course requiring students to collaboratively design a product.

Our main research questions can be summarized as follows:

1) How do the students frame space and time while discussing and performing a collaborative task?

2) How are the emerging space-time frames characterized? 


\subsection{Methods}

\subsubsection{Context and participants}

The context analysed is an interdisciplinary design course, at the Bachelor's level, held at Metropolia University of Applied Sciences in Helsinki, planned according to the principles of the trialogic learning approach (Paavola \& Hakkarainen, 2009). This approach aims at fostering learning through the collaborative development and transformation of shared objects. The course involved eight small groups, each attended by four or five students. On the first day of the course, representatives of partner companies - who acted as customers for the groups - presented authentic business problems, and each group was asked to design a product or service that addressed one of them. The course lasted 16 weeks, and the students worked for ten hours per week in a technologically rich environment involving smart boards, tablets and notebooks.

The first author of this paper presented the research to the students and asked them to volunteer as participants. Of the two groups that volunteered, only one was selected for this study because all its members were fluent in English. This group had five members, three of them Finnish (Ivy, Lenny and Rita), one Dutch (Jack) and one South African (Carl). As this was an interdisciplinary course, students came from different bachelor's degree programmes: marketing, nursing, media engineering, industrial management and IT studies.

The observed group worked on a business problem presented by a representative of an international humanitarian institution, namely the difficulty of convincing people to wash their hands carefully and frequently in order to prevent the spread of contagious diseases. The students collaborated to generate ideas about services or products addressing the problem, to meet the weekly requests of the teachers, and finally to present their product. For each sub-task, the teachers had prepared a template to guide the students' work. The most important products required by the teachers were a business plan, a user story and persona profile, a prototype of the product/service, a sales pitch presentation, and a weekly progress report. These products were created during the initial phase of the course and updated until 
a final version was produced at the end. Besides working in groups, every week the students had a steering group meeting with the teachers and their customer, and attended some lectures and plenary meetings. The students were able to organize their group work with a certain degree of autonomy, so the organization of space-time was a relevant dimension of their joint activity.

\subsubsection{Data collection and corpus of data}

For the data collection, we used qualitative methods inspired by ethnography (Hammersley \& Atkinson, 2007). We primarily used participant observation, with audio and video recordings (Goodwin, 2000). In order to follow the potential transformations in the framing of space-time and task interpretation, we collected the data during the two first weeks of the collaborative activity, during two weeks in the middle of the activity and, finally, during the last two weeks. Only the sessions in which the students worked in groups were considered; the teachers' lecturing was excluded. In total, nine sessions of group work were included in the corpus. The observations were collected by two researchers, one of them handling the camera and the other taking field notes on the general impressions of the ongoing collaboration.

The researchers generally did not intervene, but observed and changed the focus of the camera when the participants moved. To keep track of the online activity, the video records also included the smart board screen records, whenever it was used, which were synchronized with the video-audio recording for a joint analysis. Finally, we had access to a wiki page and a Dropbox folder where information and the artefacts were uploaded and updated by the students.

\subsubsection{Data analysis: Detecting the framing of space and time}

Our main data were the audio-video recordings; the other data sources were used to clarify and enrich our interpretation of the videos. The main data were catalogued (Heath et al., 2010) and organized in collections using Transana, software specifically developed for video analysis. The method we developed for the analysis of the video records involves a sequence of actions. 
First, we explored the data and defined the criteria for selecting the relevant events for in-depth analysis. We identified the following criteria based on the content of speech:

1) discourse where students a) explained to each other what they should do and how they interpreted the teachers' instructions (interpreting the task), or b) discussed the current accomplishment of the task (monitoring of the task).

2) discourse concerning a) the organization of time (e.g. the definition of the schedule); b) the organization of space (e.g. the organization of the workspaces, both in terms of physical and virtual spaces); and c) the chronotopic constraints and opportunities, that is, the constraints and opportunities related to the perceived space-time.

Second, we repeated our searches in the data to find relevant clips based on the criteria above. We selected topical episodes (Linell, 2009) as our unit of analysis. In other words, we not only selected the turns containing references to task interpretation or space-time, but all the consecutive speech turns concerning the topic at hand. We decided to use this unit of analysis because topical episodes are basic units of dialogical interaction and allow investigating the framing of space-time. One researcher conducted the searches of the data and showed the coded video clips to another researcher to discuss the coding. In cases of disagreement, a third researcher was consulted.

Third, to investigate the connection between chronotopes and task interpretation, the selected topical episodes were transcribed and qualitatively analysed. In this qualitative analysis, we interpreted the students' talk to uncover the space-time frames that were associated with the interpretation of the task. Discourse analysis (Fairclough, 2004) offers useful conceptual tools for the analysis of the space and time. Such analysis involves inferring space-time relations from discourse by considering linguistic features such as the tense, aspect and modality of verbs; adverbials; conjunctions that mark temporal relations; and phrases that mark location. Finally, some gestures, especially deictic gestures such as pointing (Goodwin, 2003), are often involved in defining space and time. By means of 
qualitative discourse analysis, we detected spatial frames (e.g. locations; positions; organization of workspaces), temporal frames (e.g. temporal sequences of actions; past, present or future events; the duration of events) and space-time constraints (e.g. time limits perceived as a constraint for the activity). In the analysis below, we do not report all the space-time frames that were present in the discourse. In order to make the excerpts readable, we have summarized the main space-time frames that we considered essential for the interpretation of the students' speech.

\section{Results}

We organize our results as follows. First, we analyse how the framing of space-time and the interpretation of the task progresses within a single session of collaboration. To accomplish this, we present the in-depth analysis of two excerpts taken during the first phase of the course and discuss a) how different interpretations of the task are negotiated within the group, and b) how the framing of space-time is connected to the interpretation of the task. Second, we analyse one excerpt from the second phase and two excerpts from the final phase to describe some further aspects of the investigated processes that emerge in these phases. Third, we discuss 1) how the framing of spacetime diachronically contributes to the trajectory of engagement within a single session of collaboration, and 2) how task interpretation and the framing of space-time evolved during the three phases of the course. Finally, we will draw our conclusions and briefly discuss the limitations of the study and future directions.

\subsection{Framing space and time at the beginning of the course}

In this section, we analyse two topical episodes from the third session of collaboration to show how the interpretation of the task and the framing of space-time were interconnected and co-developed during a single session of collaboration.

In the first excerpt (Excerpt 1), the group engaged in the task of defining the user group for the service/product they were designing. The teachers had previously described the task and presented 
the basic principles and elements for its accomplishment during an introductory lecture. After the lecture, the students moved to another room, where they sat around a table, using the smart board to visualize relevant artefacts. After about ten minutes of group work, they engaged in the following dialogue about the nature of the task and sub-tasks they needed to accomplish.

Excerpt 1: Defining the user group.

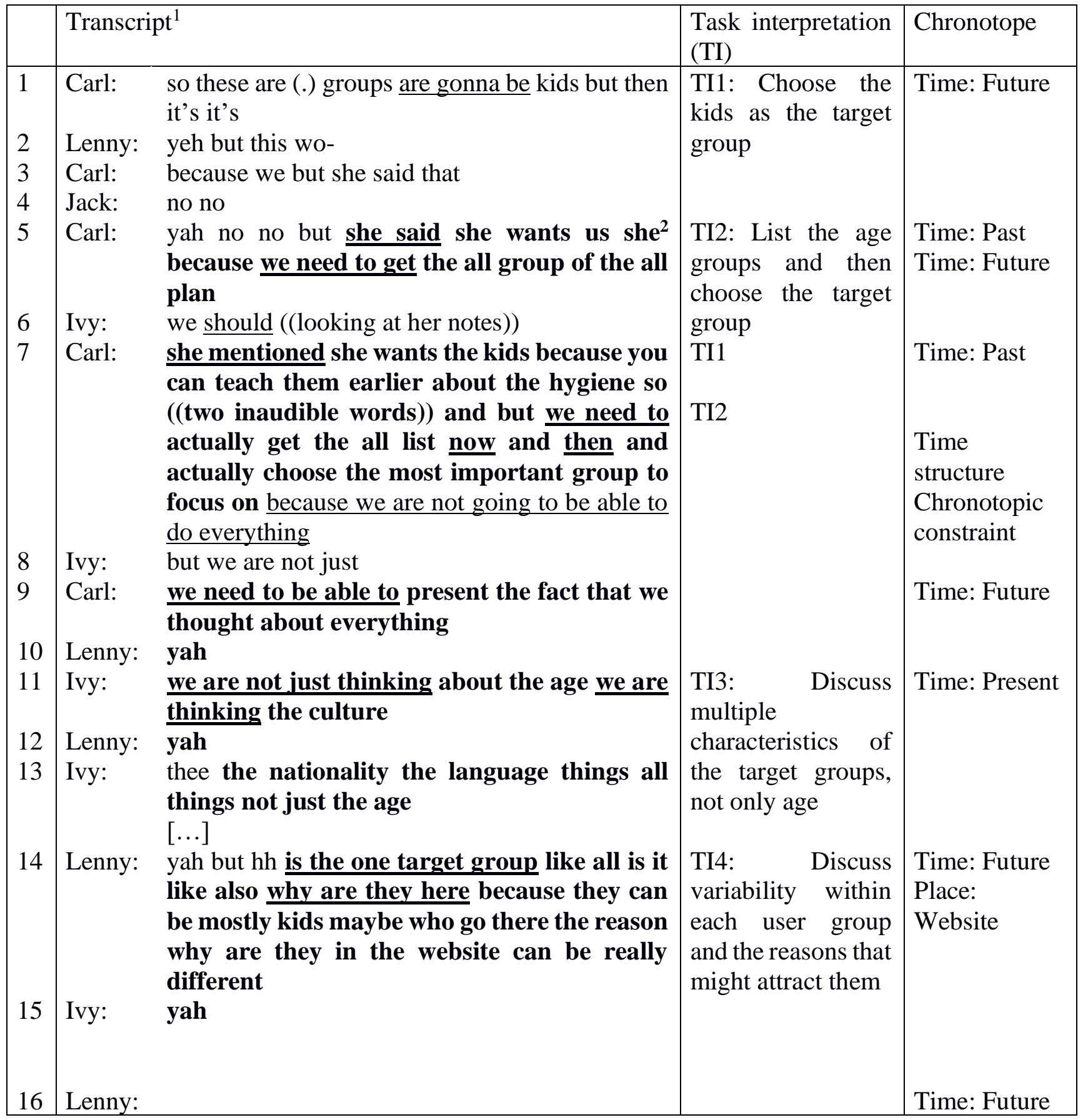

\footnotetext{
${ }^{1}$ In all the transcripts, the speech concerning a task is in bold text; the speech concerning space or time is underlined. ${ }^{2}$ The reference is to Marta, who acted as the customer of the group and suggested that children might be the target.
} 


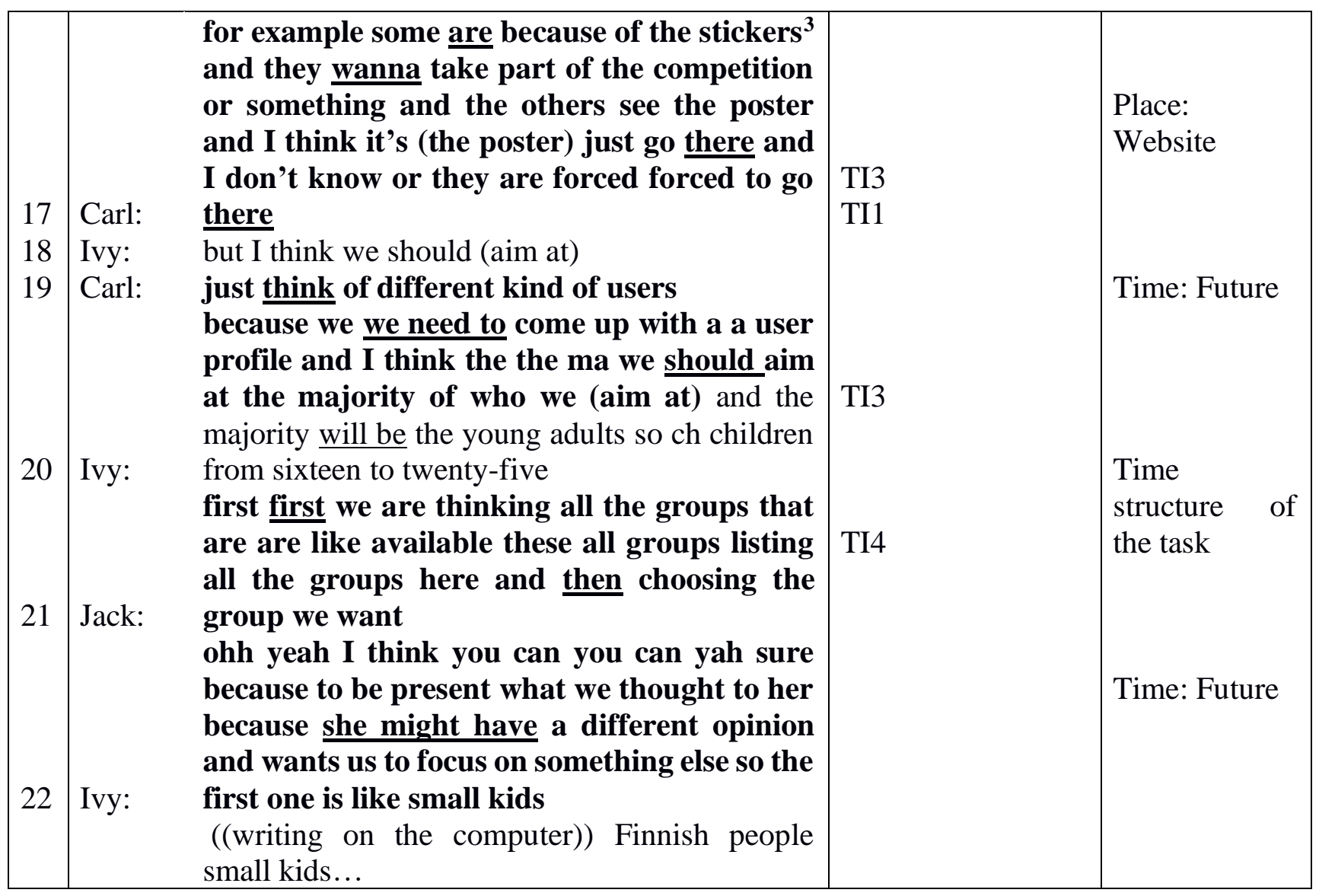

This excerpt contains a topical episode concerning the definition of the user group. The students had been provided with a set of sub-tasks that would help them structure the activity; nevertheless, the task was interpreted in multiple ways. For example, concerning the sub-task of listing the user groups, there are multiple ways in which user groups can be defined, and multiple possible criteria for organizing the list. Students may have different ideas about these issues. In particular, we noticed that some interpretations tended to expand the scope and complexity of the task (expansive interpretations) and other interpretations tended to reduce the scope of the task (reductive interpretations). In sum, we detected four interpretations:

- TI1: Focusing only on one target group, namely 'small kids' (reductive interpretation)

\footnotetext{
${ }^{3}$ Lenny here refers to a marketing strategy that they had mentioned previously which involved the use of stickers and posters.
} 
- TI2: Writing a list of possible age groups and then choosing one of them as the target group (reductive interpretation)

- TI3: Considering multiple characteristics of the user groups, such as culture or language, to choose the target group (expansive interpretation)

- TI4: Discussing the internal variability of the user groups and possible ways to reach the different people that may be included within each user group (expansive interpretation)

These task interpretations are substantially different from each other, involving different working loads and different outcomes. The discussion mainly concerns the definition of the scope of the task, which leads to the emergence of a tension between the expansive and reductive interpretations. The framing of space and time is connected to the discussion of these task interpretations in three ways:

- A future orientation: There is an active anticipation and evaluation of the potential developments of the task in the future. The students predict how the accomplishment of the task could evolve according to the current interpretation, envisioning potential trajectories in time to evaluate the different interpretations. This tendency is often recognizable thanks to the use of modal verbs and future tenses ("we are not going to"; "we should"; "she might"). Even in speech turns 14 and 16, when Lenny uses the present tense, he is actually imagining what children could do (in the future) if the group had used stickers and posters in the marketing strategy.

- The time structure of the tasks: The process of envisioning how the accomplishment of the task could evolve in the future includes defining the time structure of the activity, that is, the planning of temporally ordered sequences of sub-tasks. For example, in Excerpt 1, Carl and Ivy describe these sub-tasks using explicit references to time frames ("first [...] then"; "now [...] and then"). 
- Consideration of chronotopic constraints: The students evaluate their interpretation against the background of the perceived opportunities for and constraints on the accomplishment of the task. In Excerpt 1, this is done by Carl in turn 7. Carl points out that they should choose the most important group instead of discussing all the user groups, because "we are not going to be able to do everything". This apparently effortless conclusion by Carl is quite complex. This statement implies that Carl (a) envisioned how the activity should be carried out, how much time it would take, and what resources were needed for carrying it out, and (b) compared this envisioned course of action against the perceived space-time constraints. His statement involves two kinds of space-time: 1) the space-time that should be required for accomplishing the task, which include the workspace and the temporal duration considered necessary for the task and 2) the space-time subjectively perceived as given (in this case perceived as a constraint), which includes the workspace as it is organized at the moment and the time limits of the activity. By subjectively perceived we mean that each student might have a different view on the space-time constraints of the activity. As an example, another student might have had an expanded perception of the workspace and may then explore the internet to find some additional tools that could support and speed up the accomplishment of the expanded task (e.g. documentation regarding the typical profiles of each age group). This would involve a different perception of space, which would also transform the perception of time. Indeed, in this case, time would not be considered an insuperable constraint, as Carl considered it in the excerpt.

In sum, space-time frames were used to make sense of the task and imagine how the activity would evolve according to different interpretations. Nevertheless, the tension between the different interpretations was not resolved within the discussion taking place during Excerpt 1. Different perspectives remained and there was no agreement about how to proceed. Indeed, in Excerpt 2 a further redefinition of the activity took place. The following clip was recorded about 20 minutes after 
the previous one. The students were still working on the same task, and in the meantime, they had tried to identify all the possible user groups. In order to do so, Jack had opened a drawing application in the smart board and started representing the age groups that they were discussing (Figure 1).

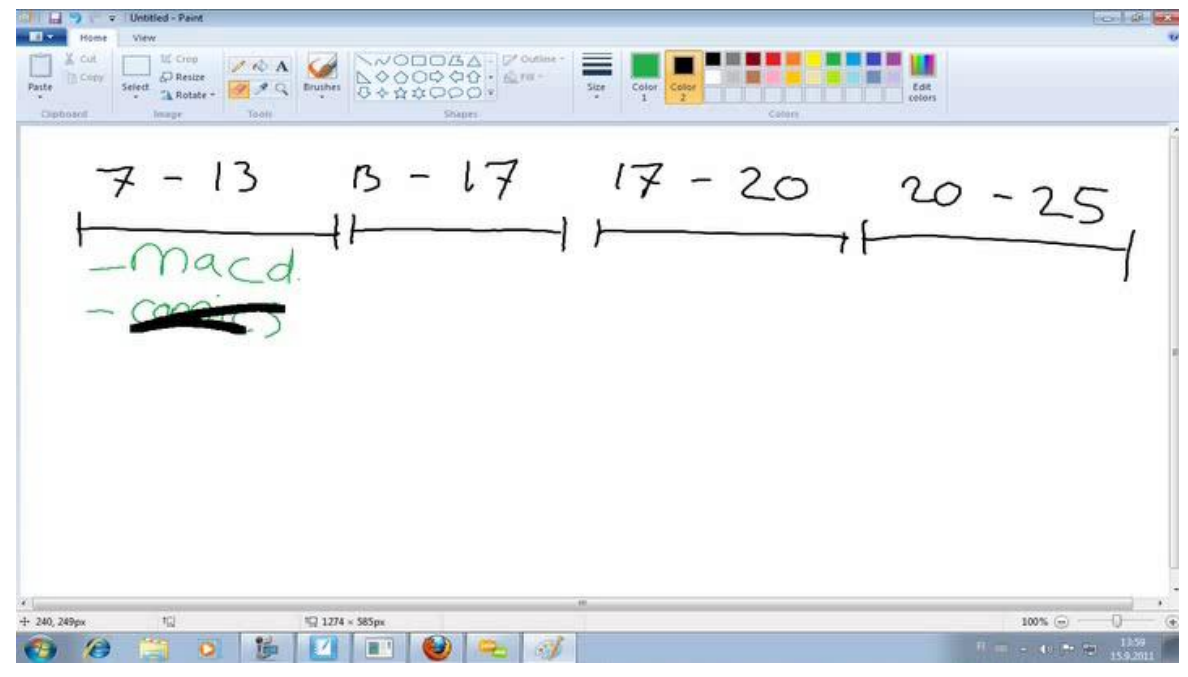

Figure 1: Screenshot of the smartboard taken at the beginning of Excerpt 2

They had defined four age groups and started discussing how they could get the attention of different kinds of people in each group (thus accomplishing TI4 in the list above). They were writing down notes concerning this discussion under the age group 7-13 ('- macd', ' - comix'). Then Ivy started wondering whether they should change their plans and progress to the following sub-task. The following excerpt presents the following discussion.

Excerpt 2: Proceeding with the user story.

\begin{tabular}{|c|c|c|c|c|}
\hline & Transcri & & Task interpretation & Chronotope \\
\hline 1 & Ivy: & $\begin{array}{l}\text { I: I don't know we should now choose } \\
\text { the one stereotypical group we want } \\
\text { like focus on so we have to get done } \\
\text { with the like user story and for the } \\
\text { user story we have to choose the group }\end{array}$ & $\begin{array}{l}\text { TI2: List the age groups } \\
\text { and then choose the } \\
\text { target group }\end{array}$ & $\begin{array}{l}\text { Present } \\
\text { Chronotopic } \\
\text { constraint } \\
\text { Time structure }\end{array}$ \\
\hline 2 & Lenny: & yah & & \\
\hline 3 & Ivy: & $\begin{array}{l}\text { so basically that's what we need to do } \\
\text { like now (..) choose the group }\end{array}$ & & Present \\
\hline 4 & Jack: & $\begin{array}{l}\text { you're not you're not gonna put down } \\
\text { a list of how we get attention to those } \\
\text { groups before you choose the group? } \\
\text { no }\end{array}$ & $\begin{array}{l}\text { TI4: Discuss variability } \\
\text { within each user group } \\
\text { and the reasons that } \\
\text { might attract them }\end{array}$ & $\begin{array}{l}\text { Future } \\
\text { Time structure }\end{array}$ \\
\hline
\end{tabular}




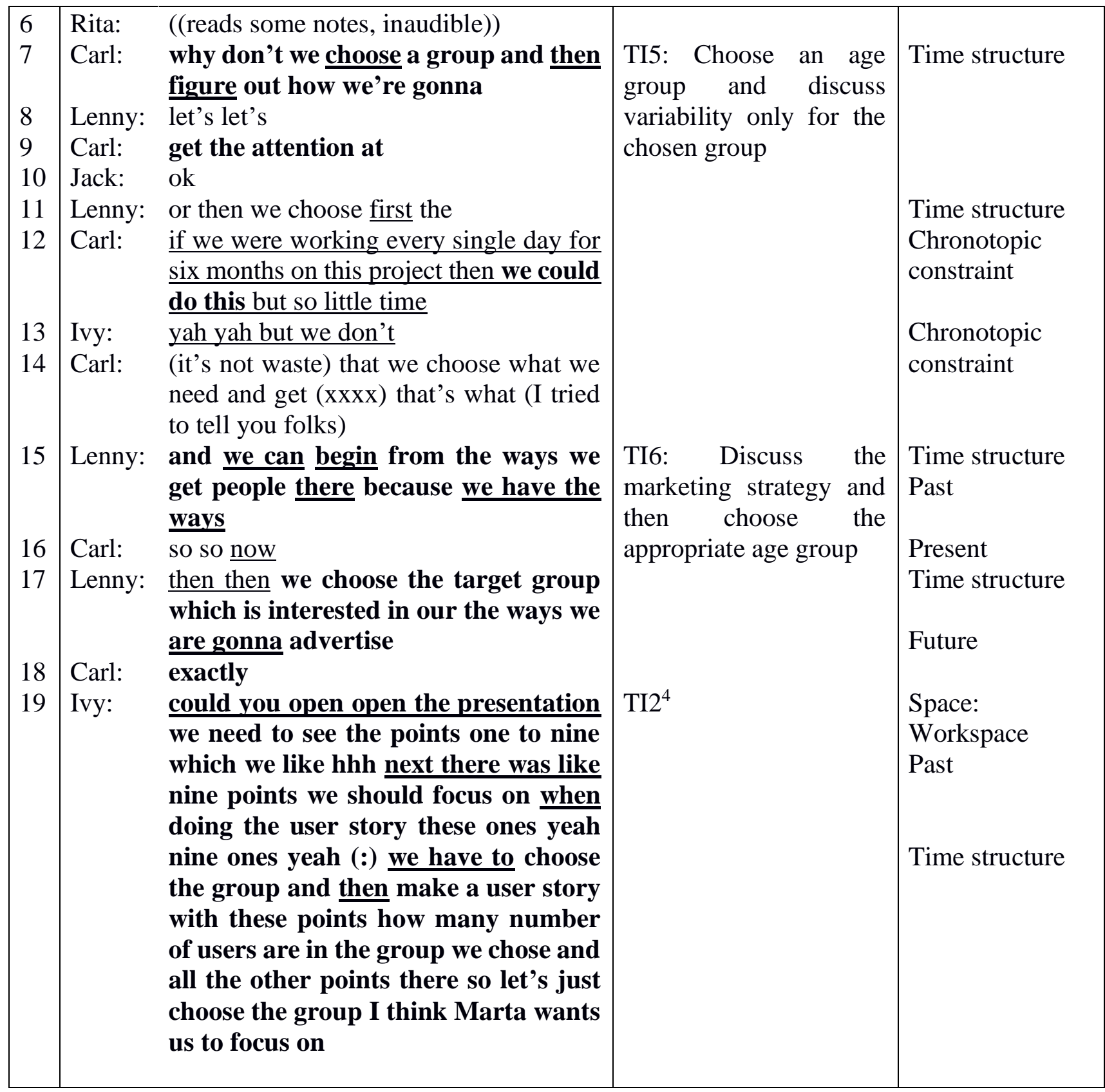

In this excerpt, the students still expressed different views on the task. While Jack believed that they had agreed about engaging in TI4, Ivy suggested that they should engage in TI2. Moreover, two new task interpretations emerged:

- TI5: Choosing an age group and discussing the possible ways of reaching the different people that may be included in it

$4 \quad$ Because of word limits, in this paper we discuss the students' interpretations concerning the sub-task leading to the choice of the user group and do not consider the sub-task of writing the user story. 
- TI6: Discussing the marketing strategy of the project and then choosing an appropriate age group

At the end of the excerpt, the students decide to engage in TI2, which is a reductive interpretation of the task.

The analysis of this excerpt involves the same aspects of the framing of space and time we discussed above, but adds a further aspect:

- The time structure of the task: For example, Jack and Lenny in turns 4 and 15 describe subtasks using explicit references to time frames;

- Consideration of the chronotopic constraints: At turn 12, Carl explicitly refers to time constraints as obstacles for the accomplishment of the expanded interpretation of the task;

- Monitoring of the task: The future-oriented envisioning of the activity also involves monitoring the outcomes of the trajectory already actualized in the past. This is evident in Lenny's attempt to propose an alternative sequence of sub-tasks: first, define the strategy to attract potential users and, second, choose the target group that fits that marketing strategy. It is implicit in his words that this organization of the activity could allow them to accomplish the task within the given time limits. Indeed, he justified his proposal by saying that they already had discussed the strategies to attract the users, so they had already completed part of the task, according to this interpretation. Lenny's argumentation here well represents the links between making sense of the task and making sense of the whole context of the activity (including its space-time dimension). He shows that his understanding of the task is grounded in his understanding of the development of their collaboration, as it is situated in the spacetime of their ongoing activity. In doing so, he takes into account both the past (what has already been done) and the future (what could/should be done; perceived time constraints; etc.). 


\subsection{Framing space and time halfway through the course}

In this section, we analyse one excerpt (Excerpt 3) taken from the second phase of the course, and we discuss how the interpretation of the task and the framing of space and time progressed in this phase. The excerpt was recorded one week before the mid-term presentation required by the teachers. During the session, the students had been working on their own laptops and talking to each other from time to time to coordinate their individual work and advise each other. The primary focus of the activity was not on face-to-face conversation, but on the virtual space of the computer. Toward the end of the morning session, they started discussing how to arrange the work for the afternoon. Within this conversation, Jack stated that he was designing a crack (to split the website into two parts) that he characterizes as "nice".

Excerpt 3: Improving the design of the website

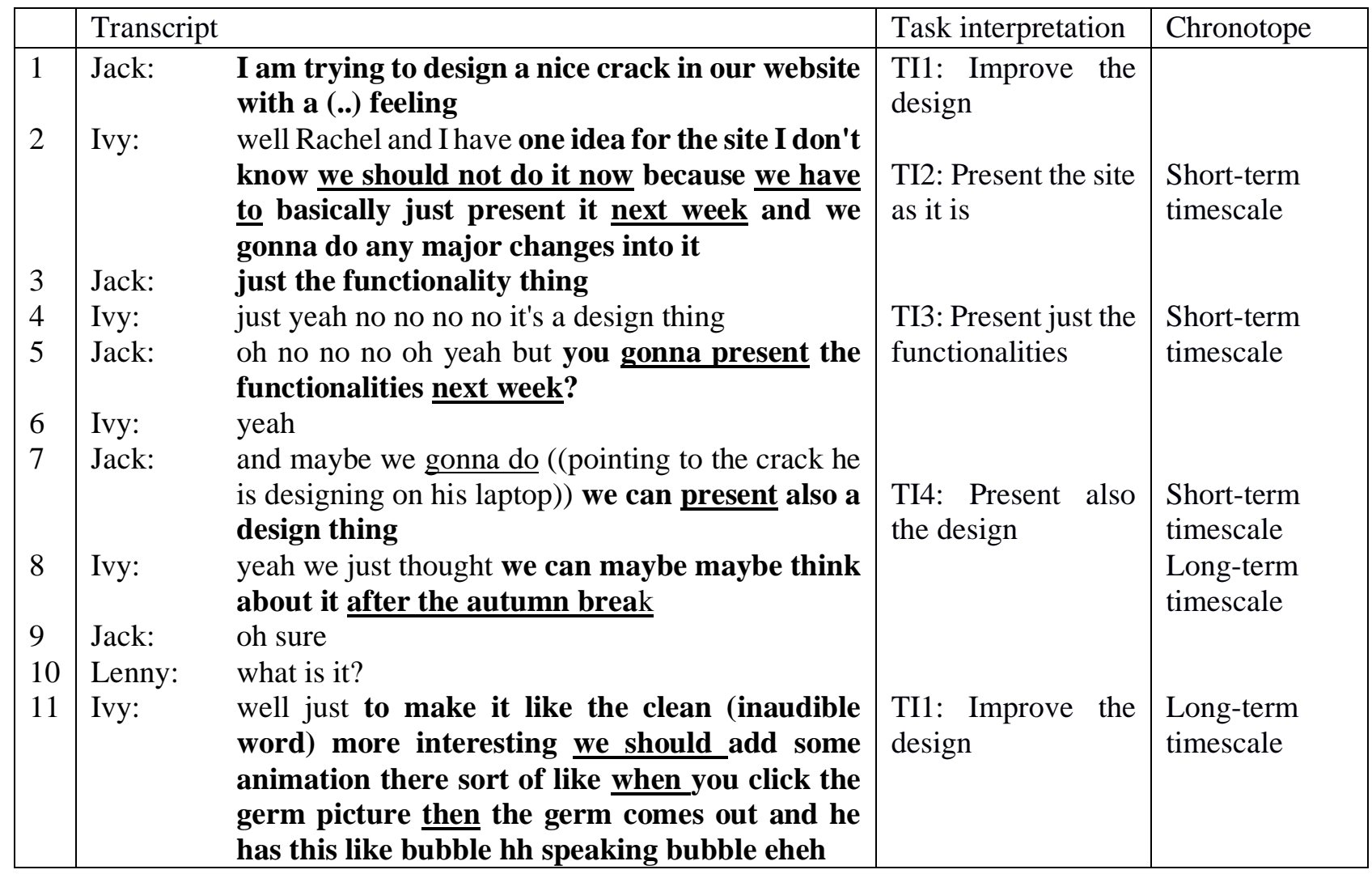


This excerpt contains some of the chronotopic dimensions discussed in the previous section. First, we detected two different time scales that define the time structure of the task in a future-oriented process: a) the timescale of the whole course (long-term timescale) and b) the timescale of the ongoing sub-task (short-term timescale). The negotiation concerning the scope of the task is strongly connected to a shift between these time scales. The excerpt begins with a discussion on the sub-task that Jack is carrying out in the present, concerning the design of the website. Then Ivy introduces a distinction between the two time scales, framing the task interpretation 1 (to improve the design of the website) as a long-term task that should not be included in the short-term task (i.e. the presentation they should give the following week). In this way, the interpretation of the task is articulated according to the temporal frames that she considers relevant for the activity. Second, in turn 2 , she considers her perceived space-time constraints as a rationale for supporting her interpretation of the task. Third, there is an ongoing monitoring of the task, that is, an evaluation of the work done in the past, which is considered sufficient for the mid-term presentation, since the students plan to present it as it is, without making any substantial changes.

We have found that the aspects of chronotope described in the previous section are also present during the second phase of the course. However, we have found some differences between the first and the second phase of the course. First, during the second phase this type of negotiation concerning the task was less frequent. The students spent more time working alone or in dyads at sub-tasks, and collective discussion was occasional, mostly used to coordinate, to support each other's work or to combine the individual work into the collective product. Second, at times a professional understanding of the task was made relevant by engagement in profession-specific sub-tasks. This professional understanding of the task became more clearly visible during the last phase of the course, when the students spent most of the time working on sub-tasks, individually or in dyads. We will discuss the professional interpretation of the task in the next section, presenting the analysis of two excerpts taken from the last phase of the course. 


\subsection{Framing space and time at the end of the course}

The following excerpt was taken from the last phase, two weeks before the end of the course. Two students - Lenny and Jack - were programming the website in the computer laboratory, while the others were engaging in different sub-tasks. These two students were both knowledgeable in computer science, and they took care of programming the website. During this session, they engaged in some very specific sub-tasks, such as the optimization of the letter spacing, in order to make the website more user friendly. This is a very specific task requiring specific procedures which nonexperts might not even realize is part of programming the website.

Excerpt 4: Optimizing the letter spacing

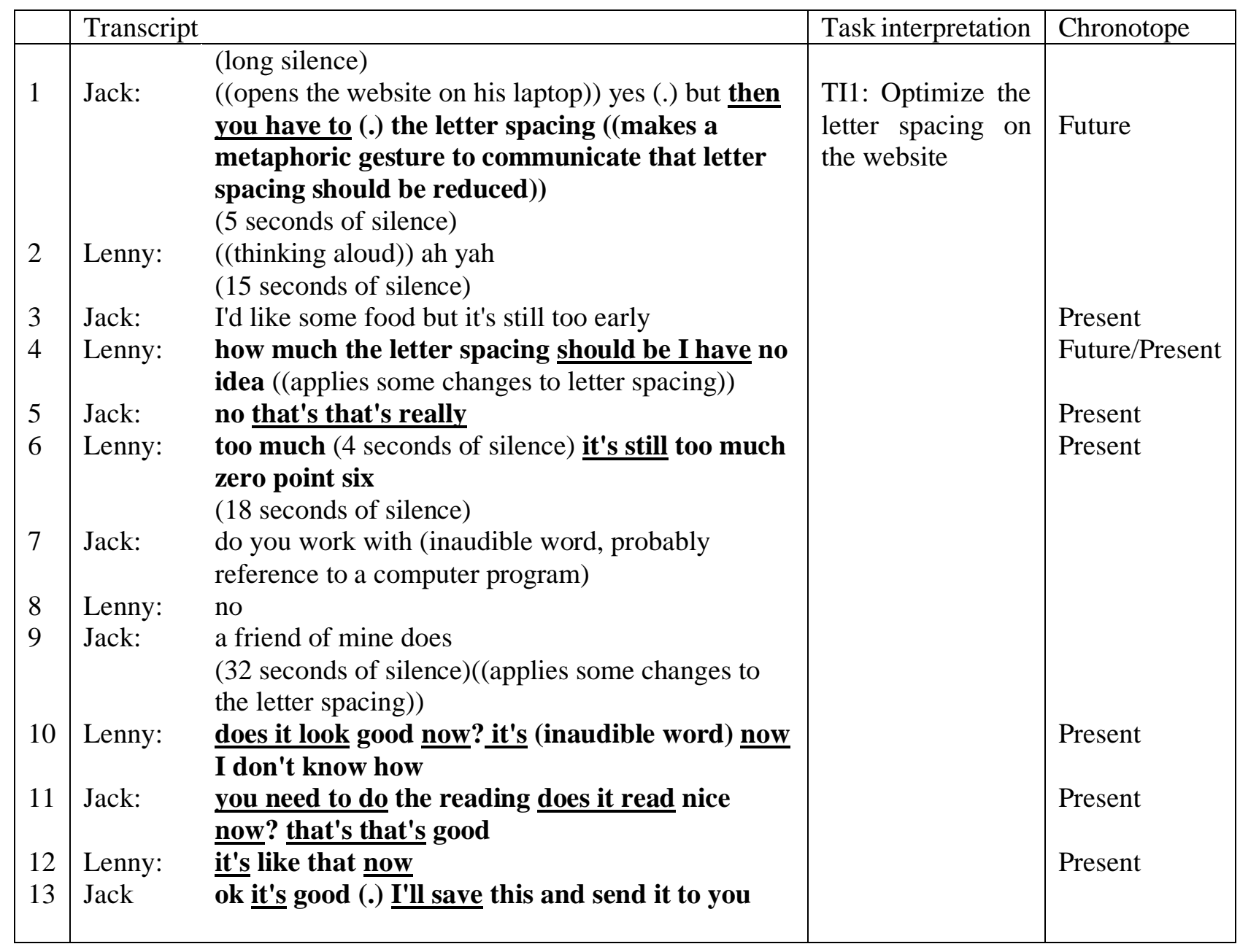


This excerpt shows that the students' interpretation of the task is dependent on their background. In this excerpt, the two students do not share the same understanding of the task. Indeed, Jack is more knowledgeable concerning this sub-task, so he has a clearer (professional) idea of what it takes to regulate the letter spacing of a website. Lenny, in contrast, is not sure about how this sub-task should be carried out and about the criteria for considering this sub-task accomplished. Consequently, Jack assists Lenny and gives suggestions to help him understand what the task involves and how to complete it. The discussion here is different from the previous excerpts, because it mainly concerns the present state of the task (monitoring the task) and how to accomplish it moment by moment. In general, in this phase it is rare to find the discursive framing of the time structure of the task in the future-oriented process that we have previously discussed. The shared frame of reference is the present.

Even though we found less negotiation about task interpretation, and the space-time frames were often reduced to cover a span of some seconds or minutes, the group had not yet reached a completely shared understanding of the task. Indeed, different perspectives of what the task entailed persisted, and these perspectives were also connected to a professional understanding of the task, which was different for each student.

The following excerpt, taken from the same session as Excerpt 4, allows this point to be discussed further. Lenny and Jack had been working on programming and improving the design of the website for about one hour and twenty minutes. After a period of silent work, they engaged in the following short dialogue.

Excerpt 5: The invisible work

\begin{tabular}{|l|l|l|l|}
\hline & Transcript & Interpretation of task & Chronotope \\
\hline Jack: $\begin{array}{l}\text { this all stuff they don't even know that it's } \\
\text { happening the other three }{ }^{5}(.) \text { just (that it } \\
\text { takes) it it costs some work }\end{array}$ & $\begin{array}{l}\text { Task interpretation 1: } \\
\text { Program and improve }\end{array}$ & Present \\
\hline
\end{tabular}

\footnotetext{
5 Jack refers to the other three members of the group.
} 


\begin{tabular}{|c|c|c|c|}
\hline $\begin{array}{l}\text { Lenny: } \\
\text { Jack: } \\
\text { Lenny: } \\
\text { Jack: }\end{array}$ & $\begin{array}{l}\text { yahh you just move them }{ }^{6} \text { there }(.) \text { it just } \\
\text { works } \\
\text { yah it did already work it hh (.) oh no this is } \\
\text { not the right one } \\
\text { ehh } \\
\text { this is the correct one ((doing something } \\
\text { related to programming)) }\end{array}$ & $\begin{array}{l}\text { the design of the } \\
\text { website }\end{array}$ & $\begin{array}{l}\text { Present } \\
\text { Space: The } \\
\text { website } \\
\text { Past } \\
\text { Present } \\
\text { Present }\end{array}$ \\
\hline
\end{tabular}

This excerpt shows that the professional understanding of the task by group members is very strong, and each student understands the sub-task differently. Indeed, Jack comments explicitly on the fact that the other students - who do not have any background in computer science - do not understand what the task of programming a website entails. Profession-specific sub-tasks, such as the optimization of the spacing of letters described in Excerpt 4, are considered by Jack and Lenny important elements of the task, but the workload for taking care of the details of the programming and improving the design of the website - in their opinion - is not fully understood by the other members of the group. In this way, the students seem to envision different space-time frames for the task. The students who are knowledgeable in programming and web design have a different understanding of the space (e.g. the virtual, physical and social spaces that might be useful for accomplishing the task) and the time (e.g. the duration and sequential structure of the task accomplished by using the appropriate software).

In addition, in this excerpt, Jack and Lenny define a social-relational space-time that excludes the other members of the group, who seem to be considered as out-group in relation to the dyad composed by technical experts. This time-space is inflected with a moral dimension of superiority (as hard workers without much recognition of their efforts). It is likely that such social-relational framing of the space contributed to hinder the reaching of a shared understanding within the group, reducing the opportunities of dialogue between the technical experts and the three other members of the group. Indeed, we observed that in the second and final phase of the groupwork, the students worked often

\footnotetext{
${ }^{6}$ Lenny refers to the fact that he had designed some interactive objects and animations for the website, and he was working with Jack to program them and finalize the design.
} 
in subgroups, with the technical experts teaming up for the programming of the websites and the others carrying out different sub-tasks (frequently in different rooms).

This framing of space-time, in its multiple dimensions, significantly contributes to the interpretation of the task and to decisions between reductive or expansive interpretations. For example, in Excerpt 3, we saw that Rachel and Ivy proposed including animations in the website, but the brief exchange between Lenny and Jack in Excerpt 5 suggests that Rachel and Ivy did not have a clear idea of the time and resources required by their proposal. Therefore, they were not aware of the space-time constraints perceived by Lenny and Jack.

\section{Discussion and conclusions}

In this article, we discuss how the social negotiation of a task is interconnected with the discursive framing of space-time during an interdisciplinary design course. Firstly, we have analysed how multiple interpretations of the task are negotiated at the beginning of the course in a future-oriented process that involves defining the time structure of the task, monitoring the task and considering space-time constraints. This process involved implicit notions of the space-time frames that are considered relevant by the students. The whole process is summarized in Figure 2. 


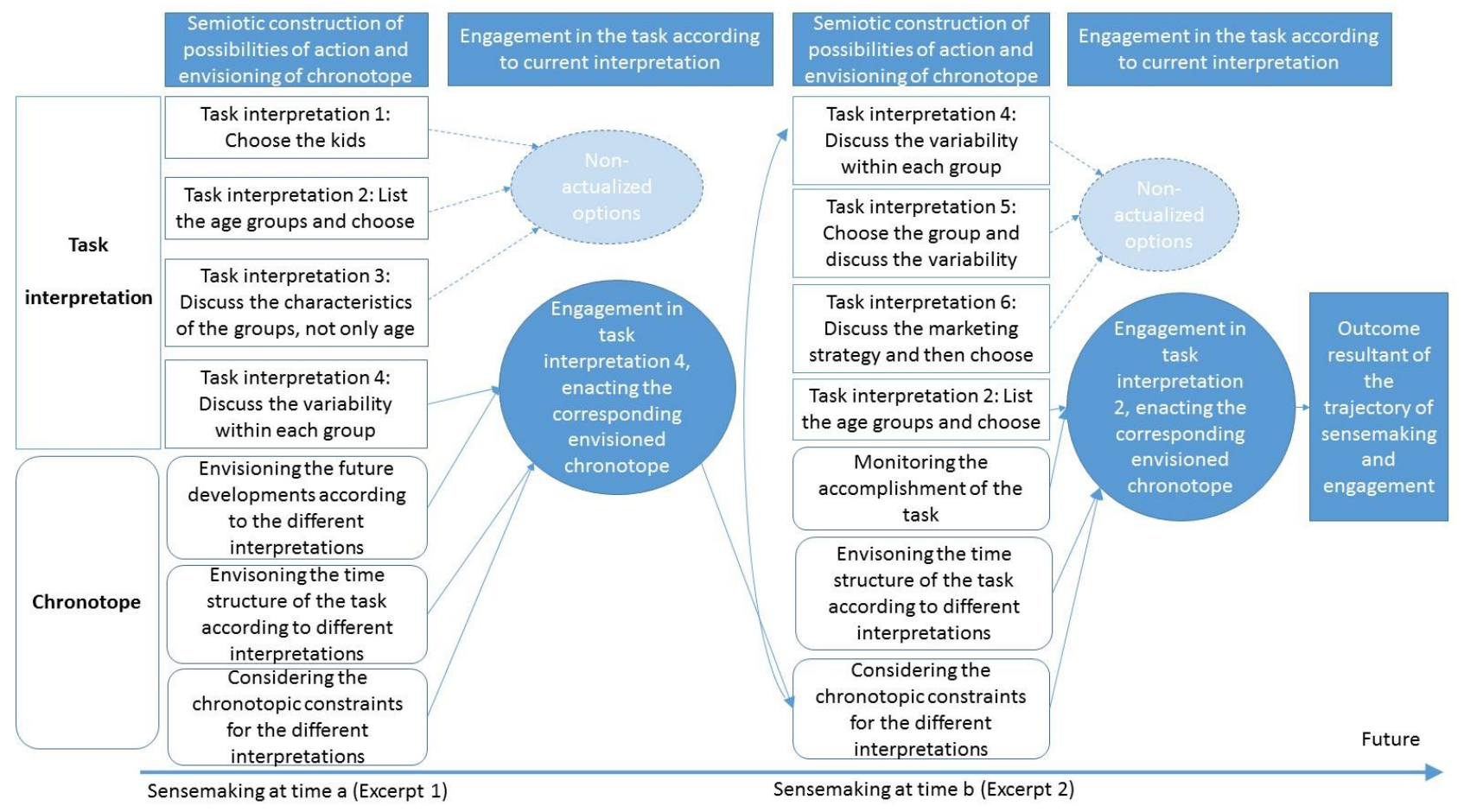

Figure 2: Visual representation of the connections between the framing of space-time and task interpretations in our analysis of excerpts 1-2

Figure 2 presents a visual summary of our analysis of the two excerpts from the first phase of the course. The figure is organized as a timeline from left to right. The first column represents the discourse on task interpretation carried out in Excerpt 1. Four different interpretations of the task emerge, and three aspects of chronotope contribute to the students' sensemaking: 1) the students envision how the task would evolve, 2) they define temporally ordered sequences of sub-tasks, and 3) they consider constraints related to the perceived space-time for the different interpretations. The second column represents the students' engagement in TI4, which took place between the two excerpts, when the students enacted the sequence of sub-tasks envisioned in speech turns 14-16 and 21 of Excerpt 1 . They engage in an expansive interpretation, probably considering Carl's concern that they are not going to be able to do everything (Excerpt 1; turn 7) unjustified. The third column represents the discussion taking place in Excerpt 2. We have found here two further interpretations of the task and further discussion of TI2, which had not yet been actualized. At the end of Excerpt 2, the students decided to engage in TI2 because some of the students perceived the constraints of the 
activity as incompatible with TI4. The fourth column represents the engagement after Excerpt 2, when the students accomplished TI2. Finally, on the right we represent the outcome of the activity, which is the result of the whole trajectory of sensemaking and engagement. Although this is a simplified representation of the process, we believe that it lends itself well to showing how the framing of space and time was intrinsically connected to the interpretation of the task and to the construction of the outcome in a recursive process. This type of future-oriented framing of space and time was present in all the phases of the course, but it gradually decreased as the group members started engaging in profession-specific sub-tasks and as less time was spent in the collective negotiation of the task. During the second and third phases, some other aspects of the space-time framing emerged: 1) a growing focus on the present moment and 2) the rising relevance of a professional understanding of the task. Table 1 represents the features of task interpretation and chronotope across the three phases of the course.

Table 1: Task interpretation and chronotope across the phases of the course

\begin{tabular}{|c|c|c|c|}
\hline & First phase & Second phase & Final phase \\
\hline Task interpretation & $\begin{array}{l}\text { Dominance of } \\
\text { collective sub-tasks } \\
\text { and negotiation of task } \\
\text { interpretation within } \\
\text { the group } \\
\text { Different } \\
\text { interpretations emerge }\end{array}$ & 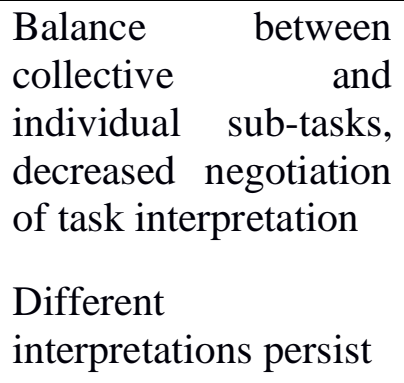 & $\begin{array}{l}\text { Dominance } \\
\text { individual of } \\
\text { and sub-tasks } \\
\text { negotiation occasional task } \\
\text { interpretation } \\
\text { Different } \\
\text { interpretations persist }\end{array}$ \\
\hline Chronotope & $\begin{array}{l}\text { Dominance of future } \\
\text { orientation } \\
\text { Chronotopic } \\
\text { constraints } \\
\text { Defining the time } \\
\text { structure of the task } \\
\text { Monitoring of the task }\end{array}$ & $\begin{array}{l}\text { Future orientation and } \\
\text { focus on the present } \\
\text { Chronotopic } \\
\text { constraints } \\
\text { Defining the time } \\
\text { structure of the task } \\
\text { Monitoring of the task }\end{array}$ & $\begin{array}{l}\text { Dominance of focus } \\
\text { on the present } \\
\text { Chronotopic } \\
\text { constraints } \\
\text { Reduced definition of } \\
\text { the time structure } \\
\text { Monitoring of the task }\end{array}$ \\
\hline
\end{tabular}


In our view, this analysis shows that the interpretation of the task is tightly connected to different space-time frames discursively articulated by the students. Grounding the envisioned, potential development of the task in space and time was an essential aspect of the students' sensemaking. Novel interpretations of the task were proposed in order for the students to adapt their activity to the perceived space-time context. In this sense, we detected a dominance of the types of space-time that we could define as - to paraphrase Valsiner - space-time as it can be and space-time as it should be, which were crucial for regulating the task interpretation.

In sum, we argue that the discursive negotiation of space and time is a further aspect of the context involved in the process of task interpretation, additional to the institutional aspects already identified by previous research (e.g. Säljö \& Windhamm, 1993; Lantz-Anderson et al., 2009). In particular, we detected an interpretative loop through which the students iteratively redefine the scope of the task and regulate the definition of their goals, based on their understanding of the activity space-time. Such an interpretive loop emerges from the envisioning of future developments of the task and the monitoring of the accomplishment of the task in the here and now, against the background of a sociocultural understanding of how the activity should develop at each stage. The students, depending also on their background, have different assumptions regarding what space-time frames are required by a given task and different perceptions of the space-time constraints of the activity. These perceptions and assumptions are used to evaluate the emerging interpretations of the task, contributing to the definition of the trajectory of engagement.

The implications of this study suggest that the discursive framing of space and time significantly contributes to the framing of the context (Engle et al., 2009). When teachers assign a task that meets specific pedagogical goals, it is important to support the process of task interpretation to ensure that the students perform the task as intended by teachers, and thus achieve the set goals. The results of our study suggest that possible differences between teachers and students in framing the space-time of the task might cause misunderstandings concerning the nature of the task. Therefore, in order to 
guide the students' interpretation of the task according to the pedagogical goals intended by the teacher, it is possible to adopt one of the following strategies:

- address potentially misleading assumptions about the space-time in which the task is embedded, in dialogue with the students;

- explore together with the students alternative - expansive - ways to conceptualize the spacetime of the activity;

- increase awareness of the implicit processes described in this article.

We are aware of the limitations of this study. The research on these processes is still at an initial phase. First, this study was carried out in a single group in a very specific context, so further research is needed to ascertain if our findings can be generalized to other settings. Our expectation is that further research might detect different modalities for the framing of space-time in different settings, but it should confirm the tight interconnection between the discursive framing of space-time and the interpretation of the task in each context. A second limitation is that our study did not involve any assessment of the students' learning. Therefore, further research is needed to verify the value of the implications of this study for development of educational practice. We expect that guiding the task interpretation through one of the strategies mentioned above might improve the adherence of the students' interpretations to the intentions of the teachers and, in turn, might support the reaching of the pedagogical goals for which the task was designed. Finally, the discussion about the space of the activity was marginal in the discourse concerning task interpretation. This could be related to the fact that the students considered the space as given or that the framing of space took place through nonverbal communication, but there is also the possibility of a methodological limitation. Indeed, the students had to book in advance the rooms in which to carry out the group work, and each room had different tools and features. Thus, they may have engaged in discussion of the space during breaks or in meeting informally before or after the scheduled meetings when the researcher was not observing. 
There is thus a need to develop research designs that make such implicit and/or informal processes visible for analysis while preserving the privacy of the research participants.

\section{References}

Bakhtin, M. (1981). The dialogic imagination. Four essays by M. M. Bakhtin. Austin, TX: University of Texas Press.

Brown, R., \& Renshaw, P. (2006). Positioning students as actors and authors: A chronotopic analysis of collaborative learning activities. Mind, Culture, and Activity, 13, 247-259.

Cole, M. (1996). Cultural Psychology: A once and future discipline. Cambridge, MA: Harvard University Press.

Duranti, A., \& Goodwin, C. (Eds.). (1992). Rethinking context: Language as an interactive phenomenon. Cambridge, MA: Cambridge University Press.

van Eijck, M., \& Roth, W. M. (2010). Towards a chronotopic theory of "place" in place-based education. Cultural Studies of Science Education, 5(4), 869-898.

Engeström, Y. (1999). Innovative learning in work teams: Analyzing cycles of knowledge creation in practice. In Y. Engeström, R. Miettinen, \& R.-L. Punamaki (Eds.), Perspectives on activity theory (pp. 377-404). New York, NY: Cambridge University Press.

Engle, R. A. (2006). Framing interactions to foster generative learning: A situative explanation of transfer in a community of learners classroom. The Journal of the Learning Sciences, 15(4), 451498.

Fairclough, N. (2004). Analysing discourse: Textual analysis for social research. London: Routledge

Goodwin, C. (2000). Practices of color classification. Mind, Culture, and Activity, 7(1 \& 2), 19-36. 
Goodwin, C. (2003). Pointing as situated practice. , in Kita, S. (Ed.), Pointing: Where language, culture and cognition meet (pp. 217-241). Lawrence Erlbaum Associates, Hillsdale, NJ, USA

Hammersley, M., \& Atkinson, P. (2007). Ethnography: Principles in practice. New York: Routledge.

Heath, C., Hindmarsh, J., \& Luff, P. (2010). Video in qualitative research. London: Sage.

Jonassen, D. H. (2000). Toward a design theory of problem solving. Educational Technology Research and Development, 48(4), 63-85.

Kirsten A. Foot (2002) Pursuing an evolving object: A case study in object formation and identification, Mind, Culture, and Activity, 9(2), 132-149, DOI: 10.1207/ S15327884MCA0902_04 Lantz-Andersson, A., Linderoth, J., \& Säljö, R. (2009). What's the problem? Meaning making and learning to do mathematical word problems in the context of digital tools. Instructional Science, 37(4), 325-343.

Linell, P. (2009). Rethinking language, mind, and world dialogically: Interactional and contextual theories of human sense-making. Charlotte, NC: Information Age Publishing.

Rajala, A., \& Sannino, A. (2015). Students' deviations from a learning task: An activity-theoretical analysis. International Journal of Educational Research, 70, 31-46.

Renshaw, Peter D. (2013). Classroom chronotopes privileged by contemporary educational policy: teaching and learning in testing times. In Phillipson, S., Kelly Y. L. Ku and Shane N. Phillipson (Ed.), Constructing Educational Achievement: A sociocultural perspective (pp. 5769). Oxon, UK: Routledge.

Roschelle, J., \& Teasley, S. D. (1995). The construction of shared knowledge in collaborative problem solving. In O’Malley, C. (Ed.) Computer supported collaborative learning (pp. 69-97). Berlin, Germany: Springer Berlin Heidelberg. 
Säljö, R., \& Wyndhamn, J. (1993). Solving everyday problems in the formal setting. An empirical study of the school as context for thought. In S. Chaiklin \& J. Lave (Eds.), Understanding practice. Perspectives on activity and context (pp. 327-342). Cambridge, MA: Cambridge University Press.

Valsiner, J. (2011) Constructing the vanishing present between the future and the past, Infancia $y$ Aprendizaje, 34(2), 141-150 